\title{
Promises and Problems of Systematic Reviews
}

\author{
Karen Rees Shah Ebrahim for the Cochrane Heart Group \\ Department of Social Medicine, University of Bristol, UK
}

The number of clinical trials published in the area of cardiology is huge. Each year, over 1,000 trials of health care interventions within cardiology are identified for inclusion in the specialist trials register of the Cochrane Library (links via www.cochrane.org). Clearly, keeping up to date with new information is extremely difficult. With the advent of systematic reviews, where similar trials are grouped to provide a summary effect estimate, the accessibility of information for busy clinicians has improved. Whilst the potential benefits of this approach are many, the methodological quality of systematic reviews published in conventional journals and their relevance to clinical practice have been raised as potential problems. This is the subject of the report by Dr. Kleist [1] in this issue of Heart Drug.

Cochrane systematic reviews differ from systematic reviews published in paper-based journals in several respects. The fundamental distinction is that authors are expected to update and amend them in light of relevant new data. There are also strict methodological guidelines to follow. Cochrane reviews are more likely to include a description of the inclusion and exclusion criteria, assess the methodological quality of individual trials and include non-English-language articles than systematic reviews published in paper-based journals [2]. These issues, particularly relating to the methodological quality of individual trials and potential sources of bias, have been highlighted in the report by Kleist [1]. Many of the problems associated with the interpretation and critical appraisal of systematic reviews are due to inadequate reporting. In an attempt to make interpretation more transparent, guidelines have recently been developed to improve the quality of reporting of systematic reviews (the QUOROM statement) [3]. Systematic reviews are certainly an advance compared to traditional 'opinion' reviews that cite evidence selectively to support a viewpoint.

Another concern is the external validity (i.e. generalisability) of findings from systematic reviews, and hence their relevance to clinical practice. Clearly, the external validity of systematic reviews is dependent on the external validity of the trials included within them. There are two main concerns. First, patients who are recruited into clinical trials are not necessarily representative. There are often age restrictions and exclusions associated with comorbidity. Findings from such trials may not be applicable to the patient population as a whole. Secondly, trials may ask questions of little relevance to clinical practice. A study by the Cochrane Heart Group [4] examined the clinical relevance of 1,008 trials of health care interventions in cardiology published in 1998. The setting where the trials were conducted was reported in only $45 \%$ of studies, and of these, only $7 \%$ were community rather than hospital based. The majority of trials were of pharmacological interventions (61.5\%). Most trials were of short duration and of relatively small size (recruiting less than 100 patients). Perhaps the most striking finding was that only half of the trials reported clinical events, and less than $4 \%$ reported quality of life as an outcome. Trials of

\begin{tabular}{ll}
\hline KARGER & ( 2001 S. Karger AG, Basel \\
Fax +41 613061234 & 1422-9528/01/0015-0247\$17.50/0 \\
$\begin{array}{l}\text { E-Mail karger@karger.ch } \\
\text { www.karger.com }\end{array}$ & $\begin{array}{l}\text { Accessible online at: } \\
\text { www.karger.com/journals/hed }\end{array}$
\end{tabular}

Dr. Karen Rees

Department of Social Medicine, University of Bristol

Canynge Hall, Whiteladies Road

BS8 2PR Bristol (UK)

Tel. +44 117928 7333, Fax +44 117928 7292, E-Mail Karen.Rees@bristol.ac.uk 
short duration with non-clinical outcomes are unlikely to influence clinical practice.

Most systematic reviews in cardiology published in conventional journals are of pharmacological interventions. This is unsurprising given that the evidence base is biased towards drug trials rather than non-pharmacological interventions; a good example of this is that currently there is just one trial of advice to cease smoking after myocardial infarction, despite the potential importance of such interventions [5]. The Cochrane Heart Group's scope of reviews is large, but we are aiming to redress the imbalance by encouraging reviews of non-pharmacological as well as pharmacological interventions. The recognition of areas where few trials are available has highlighted areas for future research. Currently, 76 reviews, completed or in progress, are registered with the Cochrane Heart Group. Of these, 36 are of pharmacological interventions, 22 of non-pharmacological interventions and the remainder are of surgical and interventional procedures.

Discrepancies have arisen between the results of systematic reviews and those from large clinical trials [6]. These discrepancies may be entirely due to publication and related biases, i.e. overestimating the treatment effect in poorly conducted meta-analyses. For example, a frequently cited review of the effects of salt restriction and blood pressure [7] which included non-randomised data demonstrated an effect size 10 times greater than several rigorous systematic reviews of only randomised controlled trials [8]. Such problems can be avoided by assessing the methodological quality of individual trials and avoiding publication bias by performing an exhaustive search of the literature [9]. In general, smaller trials are completed before larger trials, and if meta-analysed, may show a beneficial effect, making subsequent large clinical trials unnecessary and even unethical. The results of meta-analyses of small trials may also be more applicable at the population level than a single large trial, in terms of allowing stratified analyses by patient type, severity of disease, age and so on. Even in cardiology, where very large randomised controlled trials have been performed, it is unlikely that single large trials of all potentially useful treatments will be undertaken.

Finally, the estimate of treatment effect, derived from individual trials or meta-analyses, is a useful benchmark, but may bear little relationship to effectiveness at the community level. Factored into the chain from trial efficacy to community effectiveness is diagnostic accuracy, population coverage, population eligibility for treatment, patient adherence and professional competence. Unless each of these is $100 \%$, then there will be a diminution in efficacy [10]. This chain of events should certainly be borne in mind when developing clinical guidelines from the results of systematic reviews.

In conclusion, systematic reviews are the best available source of evidence to inform the development of clinical guidelines. However, critical appraisal of reports and cautious interpretation of results are essential to determine both methodological quality and external validity and hence the extrapolation of findings to clinical practice. Future challenges include continued improvement in the quality of reporting of systematic reviews, so that the process is transparent and replicable, and in conducting more pragmatic clinical trials, the findings from which, when incorporated into systematic reviews, will translate more easily into clinical practice.

\section{References}

1 Kleist P: Problems and limitations of metaanalyses within the framework of evidencebased medicine. Heart Drug 2001;1:260-267.

2 Jadad AR, Cook DJ, Jones A, Klassen TP, Tugwell P, Moher M, Moher D: Methodology and reports of systematic reviews and meta-analyses: A comparison of Cochrane reviews with articles published in paper-based journals. JAMA 1998;280:278-280.

3 Moher D, Cook DJ, Eastwood S, Olkin I, Rennie D, Stroup DF: Improving the quality of reports of meta-analyses of randomised controlled trials: The QUOROM statement. Quality of Reporting of Meta-analyses. Lancet 1999; 354:1896-1900.
4 Burke M, Beranek-Stanley M, for the Cochrane Heart Group: Randomised controlled trials in heart disease: Are they long enough and do they measure the right outcomes? Presented at the 5th South West Public Health Conference, Torquay, 2001.

5 Van Berkel TF, Boersma H, Roos-Hesselink JW, Erdman RAM, Simoons ML: Impact of smoking cessation and smoking interventions in patients with coronary heart disease. Eur Heart J 1999;20:1773-1782.

6 Egger M, Smith GD: Misleading meta-analysis. BMJ 1995;310:752-754.
7 Law MR, Frost CD, Wald NJ: By how much does dietary salt reduction lower blood pressure? 3. Analysis of data from trials of salt reduction. BMJ 1991;302:819-824.

8 Swales J: Population advice on salt restriction: The social issues. Am J Hypertens 2000;13:27.

9 Sterne JAC, Egger M, Davey Smith G: Investigating and dealing with publication and other biases; in Egger M, Davey Smith G, Altman DG (eds): Systematic Reviews in Health Care: Meta-Analysis in Context, ed 2. London, BMJ Books, 2001.

10 Smeeth L, Ebrahim S: DINS, PINS, and things clinical and population perspectives on treatment effects. BMJ 2000;321:952-953. 